\title{
RESIDUOS SÓLIDOS EN VIVIENDAS DE INTERÉS SOCIAL
}

\section{SOLID WASTE IN SOCIAL INTEREST HOUSING}

\author{
Luz Petro Falón ${ }^{1}$, Mónica Ocampo Rivero ${ }^{2}$, Brigitte Rodríguez M. ${ }^{3}$, Yuleidis Polo A. ${ }^{4}$ \\ Recibido para publicación: Septiembre 19 de 2017 - Aprobado para publicación: Noviembre 29 de 2017
}

\begin{abstract}
RESUMEN
Objetivo: Determinar el conocimiento y actitud, acerca del manejo de los residuos sólidos domiciliarios, que tienen los habitantes de unas viviendas de interés social del municipio de Montería. Materiales y métodos: Estudio descriptivo, cuantitativo, transversal,coninformaciónrecolectada mediante aplicación de un cuestionario, que evaluó aspectos relacionados conel conocimientoyactitud de las personas acerca del manejo de los residuos sólidos domiciliarios. Se realizó en 24 torres de apartamentos de viviendas de interés social; se tomó una muestra aleatoria de 175 apartamentos, en donde se aplicó un cuestionario a los jefes de hogar para la recolección de la información, la cual fue recolectada en físico y procesada en SPSS. Resultados: Los hallazgos indican que el $68 \%$ de los jefes de hogarmanifiestan que tienen conocimiento de algunas técnicas de reciclaje; el $60 \%$ refieren que no separan los residuos; el $70 \%$ no separa los desechos tecnológicos; $65,7 \%$ no tiene claridad acerca de cuáles son los llamados residuos sólidos; el $63,5 \%$ no conoce el código de colores que permite una clasificación adecuada de los residuos. Conclusión: En las viviendas objeto de estudio, es necesario implementar estrategias para mejorar el conocimiento delos habitantes que contribuya a una adecuada separación en la fuente de los residuos sólidos domiciliarios, para favorecer la reducción en la producción de basura y el desarrollo de una conciencia del cuidado al ambiente.
\end{abstract}

Palabras clave: residuos domiciliarios, viviendas de interés social, separación en la fuente.

\begin{abstract}
Objective: Determine the knowledge of and attitude toward the management of solid household waste that the inhabitants of social housing have in the municipality of Monteria. Materials and methods: This was a descriptive, quantitative, and cross- sectional study with information collected through the application of a questionnaire, which evaluated aspects related to the knowledge of and attitude of people toward the management of solid household waste. The study was carried out in 24 apartment towers of social-interest housing. A random sample of 175 apartments was chosen, and a questionnaire was administered to the heads of households for the collection of the information, which was collected in physical form and processed in SPSS. Results: The findings indicated that $68 \%$ of heads of households have knowledge of some recycling techniques; $60 \%$ reported that they do not separate the waste; $70 \%$ of them did not separate technological waste; $65.7 \%$ of the respondents were not clear about what is called solid waste; and $63.5 \%$ of them did not know the color code that allows an adequate classification of waste. Conclusion: In the homes under study, it is necessary to implement strategies to improve the knowledge of the inhabitants that contribute to an adequate separation in the source of the household solid waste to favor the reduction in the production of garbage and the development of an awareness of the care for the environment.
\end{abstract}

Keywords: household waste, social housing, separation at source.

${ }^{1}$ Enfermera M.Sc en Epidemiología. Profesora Facultad Ciencias de la Salud, Universidad de Córdoba Montería, Colombia. Correspondencia: e-mail:Inpetro@correo.unicordoba.edu.co.

${ }^{2}$ Enfermera M.Sc en Salud Pública. Profesora Facultad Ciencias de la Salud, Universidad de Córdoba Montería, Colombia.

${ }^{3,4}$ Administradora en Salud. Universidad de Córdoba 


\section{INTRODUCCIÓN}

La generación de los residuos sólidos es consecuencia del trabajo realizado por el hombre en una sociedad que consume y crea gran cantidad y variedad de residuos procedentes del quehacer diario $(1,2,3)$, el manejo poco eficiente que se le realiza a éstos y los impactos negativos que genera, depende en gran medida de la población y del proceso de urbanización. Es un tema de importancia mundial, donde se han planteado soluciones desde diversas disciplinas, que guarda una relación directa con el aumento de la población y desarrollo económico $(4,5,6)$.

El artículo 2 del decreto 2981 de 2013 del Ministerio de Vivienda define como residuo sólido domiciliario a "cualquier objeto, material, sustancia o elemento principalmente sólido resultante del consumo o uso de un bien en actividades domésticas y que puede ser aprovechable o no aprovechable" (7).

De acuerdo con el Banco Interamericano de Desarrollo, aunque existe un mayor nivel de conciencia tanto en las autoridades como en la sociedad en mejorar la disposición final de residuos sólidos, ésta sigue siendo inadecuada, lo que puede deberse a la falta de innovación y avance en aspectos de la integralidad del servicio de aseo como son la implementación de prácticas de reducción, rehuso y reciclado. Al respecto concluye que aunque no existen estudios epidemiológicos que demuestren la relación causal entre residuos sólidos y enfermedades, la presencia de los residuos se convierte en el habitat de muchos vectores transmisores de enfermedades (8).

Según la Asociación Colombiana delngeniería Sanitaria y Ambiental, ACODAL, en Colombia se producen aproximadamente unas 31.000 toneladas diarias de residuos sólidos de los cuales cerca de 26.350 , es decir, el $85 \%$ son aprovechables y por no ser reciclados están copandola capacidad de los rellenossanitarios. Al año son cerca de 11.315.000 toneladas de residuos de los cuales 9.617 .750 se descartan como basura cuando pudieran ser recuperados y aprovechados de diferentes formas de acuerdo a su composición. Lo que refleja la ausencia de conocimiento y desarrollo de una cultura de aprovechamiento de residuos desde la fuente y evidencia el bajo impacto de las políticas en materia ambiental específicamente para residuos sólidos domiciliarios (9).

Los resultados de la encuesta realizada por el Departamento Nacional de EstadísticasDANE-, la cobertura promedio del servicio de aseo en los últimos cinco años han sido de 96,5\% para el área urbana y de 20,4\% para la zona rural, con un incremento del 2,54 y del $13,61 \%$ respectivamente entre los años 2008 a 2012 (6). Sin embargo, no existe un proceso adecuado de separación en la fuente que facilite el aprovechamiento de los residuos sólidos, reduzca los volúmenes de desechos que van a los rellenos y contribuya a la preservación del ambiente.

En Colombia, mediante el decreto 2981 de 2013, se encuentra reglamentado la prestación del servicio público de aseo, teniendo como finalidad fortalecer procesos de planeación en la gestión integral de residuos sólidos, fundamentado en la programación del servicio y la gestión del riesgo en salud y ambiente, garantizando una recolección selectiva y estaciones de clasificación y aprovechamiento, sin embargo no se ha desarrollado una estrategia educativa que garantice una cultura del aprovechamiento, clasificación en la fuente y preservación del ambiente desde el hogar (10).

Con relación a las políticas de fortalecimiento de viviendasparala población deescasos recursos, se construyeron en Colombia viviendas de interés social, entendidas como aquellas que se desarrollan para garantizar el derecho a la 
vivienda de los hogares de menores ingresos, teniendo en cuenta, entre otros aspectos, las características del déficit habitacional, las posibilidades de acceso al crédito de los hogares, las condiciones de la oferta, el monto de recursos de crédito disponibles por parte del sector financiero y la suma de fondos del Estado destinados a los programas de vivienda $(11,12)$.

Estas viviendas de interés social en la ciudad de Montería, fueron entregadas gratuitamente y construidas en conjuntos habitacionales con múltiples unidades domiciliarias, ubicadas en torres de edificios, para favorecer poblaciones desplazadas víctimas del conflicto armado o de fenómenos naturales, con lo cual se pretende reducir su problemática social y de salud. Teniendo en cuenta que es una población numerosa, diversa en cultura y costumbres, cuyo nivel educativo por lo general es bajo y sin educación sobre el tema, cabe anotar que este proceso de urbanización exige una adecuada adaptación a la convivencia sana y una modificación dehábitosy costumbres, entrelos que se encuentran, la adecuada disposición de residuos sólidos y la utilización de los recursos naturales y servicios públicos. En el Municipio de Montería, el sistema de recolección tiene una cobertura de aproximadamente 75\% y cuenta con un sitio de disposición final insuficiente, efectuándose esta actividad en tiraderos a cielo abierto los cuales no cuentan con infraestructura y métodos que permitan prevenir la contaminación del aire, suelo y agua.

Teniendo en cuenta la situación que en materia de recolección, manejo y disposición final de residuos sólidos enfrenta la ciudad, así como también la necesidad de contar con estudios actuales que permitan abordar esta problemática, se planteó como objetivo determinar el conocimiento y actitud acerca del manejo de los residuos sólidos domiciliarios que tienen los habitantes de unas viviendas de interés social del municipio de Montería como un primer paso para obtener información aproximada del comportamiento del evento en un contexto social de múltiples características especiales.

\section{MATERIALES Y MÉTODOS}

\section{Tipo de estudio}

Descriptivo, cuantitativo, transversal, durante el periodo correspondiente a los meses de Julio a Diciembre del año 2015.

\section{Población de estudio}

El estudio se realizó en 24 torres de 20 apartamentos cada uno.

\section{Muestra}

Se tomó una muestra aleatoria de 175 apartamentos, en donde se aplicó un cuestionario a los jefes de hogar, la recolección de la información se calculó con una confianza del $90 \%$, un valor de $\mathrm{p}$ de 0,05 y un error permisible de 0,05. El muestreo fuealeatorio simple con reemplazo, en el cual si un jefe de hogar no estaba al momento de la visita se encuestaba al de la unidad domiciliaria siguiente, previa explicación y firma del consentimiento informado.

\section{Criterios de inclusión}

Familias residentes en la urbanización La Gloria Uno de la ciudad de Montería. Tener un adulto mayor de 18 años para dar información del cuestionario.

\section{Criterios de exclusión}

Adultos con discapacidad mental. Residentes de menos de un mes en el conjunto de viviendas.

Procesamiento y análisis de la información Se aplicó un cuestionario denominado "Encuesta residuos sólidos en tu vida" disponible en el sitio web de la Universidad de Santander, adaptado de acuerdo a los objetivos planteados. Estructurado en aspectos fundamentales relacionados con el 
conocimientoacerca delmanejo delos residuos en la vivienda, actitud personal frente a ellos y conocimiento de normas sobre el tema. Las opciones de respuesta consideraron cinco opciones o niveles de acuerdo o desacuerdo con las afirmaciones propuestas. Para la presentación de los resultados las respuestas se reagruparon en dos opciones: De acuerdo y en desacuerdo. La información fue digitada en Excel, consolidada en tablas de frecuencias absolutas y relativas en proporciones usando el software estadístico SPSS de libre versión.

\section{RESULTADOS}

Con relación al conocimiento y actitud sobre el manejo de residuos sólidos en la vivienda se encontró, que el $68 \%$ de los jefes de hogar consideranquese tieneinformaciónypractican técnicas de reciclaje; el 65\% expresó tener varios depósitosdebasurasacordeaunaclasificación, figura 1 ; el 60\%, tienen recipientespara realizar la clasificación de los residuos sólidos; el 57\% dijeron que las personas que conviven con ellos practican la separación adecuada de residuos sólidos. El 97\% consideraron tener en el hogar un lugar apropiado para acumular los residuos sólidos antes de ser depositados en el depósito comunitario.

En cuanto al manejo de residuos sólidos y la actitud personal se encontró que: el $60 \%$ de los jefes no separa los residuos sólidos al momento de depositarlos en la basura, el 70\% no separa los desechos de la tecnología (cartuchos de tinta, pilas de celulares, entre otros) figura 1 , del resto de residuos sólidos.

Además, se evidenció que el 53\% deposita la basura en los recipientes destinados para la envoltura que contiene el alimento que consumen. El $65,7 \%$ no tiene claro cuáles son los llamados residuos sólidos, el 63,5\% no Identifica los colores de los recipientes que conllevan a una clasificación adecuada de residuos sólidos. El 96,6\% le molesta

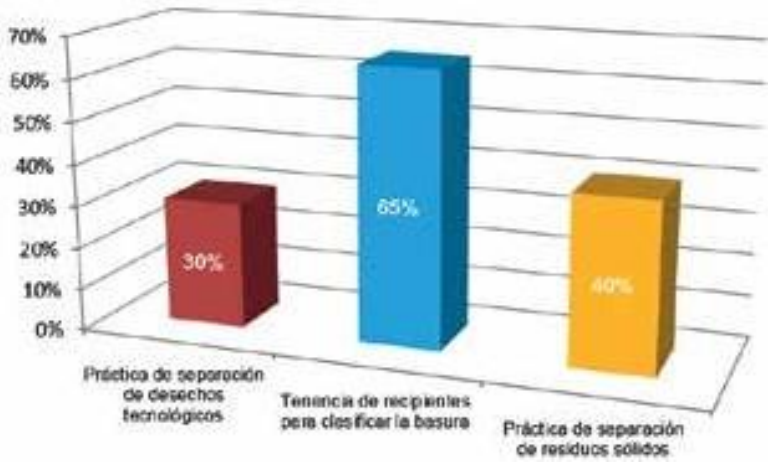

Figura 1. Práctica de separación de los residuos.

observar que en el lugar donde se encuentran haya residuos sólidos tirados. El $84 \%$ de los encuestados se considera uno de los generadores de residuos sólidos.

Acerca del conocimiento de normas sobre manejo de residuos en el hogar, como se muestra en la figura 2, el 66\% de los encuestados manifestaron no conocer la normatividad relacionada con el tema.

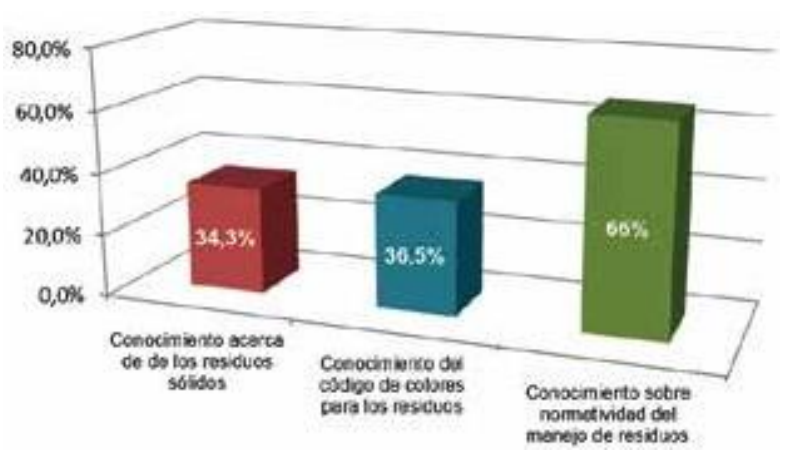

Figura 2. Conocimiento acerca del manejo de residuos domiciliarios

\section{DISCUSIÓN}

Jaramillo y Zapata (13), y otros autores (14), han expresado que existe una relación directa entre residuos sólidos, aumento dela población y desarrollo económico, por lo tanto, la fuerte relación de estos aspectos hace que el planteamiento de estrategias que permitan 
minimizar los impactos negativos sea complejo. Además, según el Banco Interamericano de Desarrollo y la Organización Panamericana de la Salud, la generación de residuos sólidos urbanos depende de la población y del proceso de urbanización (15). Al respecto, en la población sujeto de estudio se identificó que la mayoría de los encuestados se considera generadores de residuos sólidos.

Según un estudio realizado por la "Universidad Católica de Valparaíso", La caracterización de los residuos sólidos domiciliarios tiene su importancia en cada una de las fases de la gestión integral de los residuos sólidos (generación, almacenamiento, recogida, transporte, tratamientos intermedios y eliminación final) (16), en los resultados obtenidos se encontró, que en la mayoría de las viviendas no se realiza una separación de los residuos sólidos y no sólidos en el momento de depositarlos en la basura, además no separan los desechos de la tecnología del resto de residuos sólidos, no tienen claro cuáles son los llamados residuos sólidos, y tampoco identifican loscolores que conllevan a una clasificación adecuada de residuossólidos. Porlotanto los residuos sólidos domiciliarios que se generan en su mayoría son desaprovechados porque no son utilizados para su recuperación (17), además existe un desconocimiento acerca de los procedimientos o forma de hacerlo (18), así mismo con base en los resultados encontrados se pudo determinar quelamayoríadelosencuestadosmanifestaron no conocer la normatividad relacionada con el tema.

\section{CONCLUSIONES}

1. Se pudo determinar que en una población diversa en su procedencia urbana y rural, desplazados víctimas del conflicto armado o de fenómenos naturales, diversa en cultura y costumbres, cuyo nivel educativo por lo general es bajo y sin educación sobre el tema, la oportunidad de tener una vivienda de interés social, los ha inducido a cumplir con normas de convivencia que fomenten la limpieza del hogar y la adecuada disposición de residuos sólidos, proceso de urbanización que exige una adecuada adaptación a la convivencia sana y una modificación de hábitos y costumbres, entre los que se encuentran, la adecuada disposición de residuos sólidos, para lo cual el $65.7 \%$ de los encuestados manifiestan que no tienen claridad acerca de cuáles son los llamados residuos sólidos.

2. Aunque el $68 \%$ de los jefes de hogar refieren que tienen conocimiento de algunas técnicas de reciclaje, existe una carencia de prácticas de algún mecanismo para aprovechar los residuos sólidos, por lo que se hace necesario educar y concientizar a la ciudadanía acerca de la importancia de reutilizar o reciclar los residuos sólidos lo que genera un valor ambiental, reduciendo los volúmenes de residuos sólidos $\mathrm{y}$ beneficios económicos.

3. Aunque la urbanización cuenta con almacenamiento temporal de los residuos sólidos, el $63,5 \%$ de los encuestados manifestaron que no conocen el código de colores, lo que indica que no se está realizando una separación en la fuente adecuada.

\section{RECOMENDACIONES}

De acuerdo con los objetivos planteados, los resultados y las conclusiones, el grupo investigador propone realizar educación y enseñarle a la comunidad los mecanismos de aprovechamiento de los residuos y la adecuada separación en la fuente; actividades que deben realizarse en las instituciones educativas del área de influencia.

Además, las autoridades competentes deben crear mecanismos para facilitar laseparación en la fuente. 


\section{CONFLICTO DE INTERÉS}

Los autores declaran no tener conflicto de interés.

\section{REFERENCIAS}

1. Organización Panamericana de la Salud y Ministerio de Medio Ambiente de Colombia. Análisis Sectorial de Residuos Sólidos en Colombia. Programa de Gestión Urbana/Ministerio de Salud de Colombia. Abril, 1996

2. Escamirosa M, Lorenzo F, Carpio Penagos, Carlos Uriel del, Castañeda Nodasco, Gabriel. Manejo de los residuos sólidos domiciliarios en la ciudad de Tuxtla Gutierrez Chiapas [Internet]. México, D.F.: Plaza y Valdés, S.A. de C.V.; 2001.

3. Flores López, Jorge Luis. Proyecto L1C2-120 FPA: “Implementación del sistema de manejo integral de residuos sólidos urbanos en el distrito de Las Lomas [Internet]. Lima: B Municipalidad Distrital de Las Lomas; 2012.

4. Ministerio de Vivienda, Ciudad y Territorio. Marco de gestión ambiental y social. Colombia, Agosto de 2014. Disponible en http://www.minvivienda.gov.co/

Residuos\%20Solidos/Marco\%20de\%20

Gesti\%C3\%B3n\%20Ambiental\%20y\%20

Social\%202014.pdf

5. Euformación Consultores S L. editor. Recogida y transporte de residuos urbanos o municipales (2a. ed.) [Internet]. Andalucía: IC Editorial; 2017.

6. Sadhwani Alonso, José Jaime. Gestión y tratamiento de residuos I [Internet]. Las Palmas de Gran Canaria: Universidad de Las Palmas de Gran Canaria. Servicio de Publicaciones y Difusión Científica; 2015.

7. Decreto 2981 de 2013 Ministerio de Vivienda Ciudad y Territorio,
Republica de Colombia. Disponible en http://wsp.presidencia.gov.co/ Normativa/Decretos/2013/Documents/ DICIEMBRE/20/DECRETO\%202981\%20 DEL\%2020\%20DE\%20DICIEMBRE\%20 DE\%202013.pdf

8. Terraza H. Manejo de Residuos Sólidos Lineamientos para un Servicio Integral, Sustentable e Inclusivo. Banco InterAmericano de Desarrollo Departamento de Infraestructura y Medio Ambiente NOTA TECNICA No. IDB-TN-101 disponible en https: / / publications.iadb.org/bitstream/ handle / $11319 / 2850 /$ Manejo\%20 de\%20Residuos\%20S\%C3\%B3lidos.. pdf?sequence $=1$

9. Forero S, Ochoa M. Situación de la disposición final de residuos sólidos en Colombia - diagnostico, 2009. Disponible en http://www.superservicios.gov.co/ content/download/1259/16220

10. Departamento Administrativo Nacional de Estadística (DANE) (2015): Información general. Disponibleen: www.dane.gov.co

11. Ministerio del medio ambiente vivienda y desarrollo territorial. Guías de Asistencia Técnica para Vivienda de Interés Social. Colombia. Disponible en http://www. minvivienda.gov.co/Documents/guia_ asis_tec_vis_1.pdf

12. Banco Inter-Americano de Desarrollo Departamento de Infraestructura y Medio Ambiente. Nota técnica No. IDB-TN-101. Disponible en https://publications. iadb.org/bitstream/handle/11319/2850/ Manejo\%20de\%20Residuos\%20 S\%C3\%B3lidos..pdf?sequence=1

13. JaramilloG, Zapata L. aprovechamientode losresiduossólidosorgánicosen Colombia. Universidad de Antioquía, 2008. 
14. Olaya J, Ippolito K, Moreno G, González V, Klinger R, Parra CM, \& Marmolejo L. (2013). Asociaciones entre la composición socioeconómica familiar y la generación urbana de residuos sólidos domiciliarios/ associations between familial socioeconomic composition and urban residential solid waste generation. Revista ElA, 10(20), 127-137. Retrievedfromhttps://ezproxyucor.unic ordoba.edu.co:218/docview/14982363 40 ? accountid $=137088$

15. Banco Interamericano de Desarrollo y de la Organización Panamericana de la Salud. Situación del Manejo de los Residuos Sólidos Municipales en América Latina y el Caribe, ALC, Julio, 1997.

16. Pontificia Universidad Católica de Valparaíso. Facultad de Ingeniería. Informe final. Estudio caracterización de residuos sólidos domiciliarios en la región metropolitana. Valparaíso, Marzo 2006.
17. Mercurio E. Reciclaje de residuos domiciliarios 2014, septiembre 14. El Mercurio Retrieved from https:// ezproxyucor.unicordoba.edu.co:218 $6 /$ docview $/ 1562458180$ ?accountid=13 7088

18. Marmolejo Lf, Oviedo ÉR, Jaimes JC, \& Torres P. Influencia de la separación en la fuente sobre el compostaje de residuos sólidos municipales 2010. Agronomía Colombiana, 28(2), 319-328. Retrieved from https:// ezproxyucor.unicordoba.edu.co:218 6/docview $/ 1677585160$ ?accountid=13 7088. 